Trauma Berufskrankh 2004 - 6 [Suppl 3] : S344-S350 DOI 10.1007/s10039-003-0724-1

Online publiziert: 15. April 2003

(c) Springer-Verlag 2003

A.Prokop · A.Jubel · U.Hahn · K.E. Rehm

Klinik und Poliklinik für Unfall-, Hand- und Wiederherstellungschirurgie,

Klinikum der Universität zu Köln, Köln

\title{
Speichenköpfchenbrüche: Wann soll wie operiert werden?
}

Mason-I-Frakturen werden konservativ frühfunktionell behandelt. Nach kurzer Ruhigstellung (3-7 Tage) im Oberarmgips wird eine gipsfreie Nachbehandlung mit Physiotherapie und einer Entlastung über 6 Wochen angeschlossen [10]. Eine Punktion des Gelenkergusses bei schmerzhafter Kapselspannung bewirkt eine sofortige Linderung der Schmerzen. Als Punktionsort wird der Mittelpunkt einer Verbindungslinie zwischen Olekranonspitze und Epikondylus radialis empfohlen.

Die Mason-II- und -III-Frakturen werden operiert. Je nach Autor werden rekonstruktive oder resezierende Maßnahmen mit und ohne prothetischen Ersatz vorgeschlagen $[1,4,5,13,14,21]$.

Bei den rekonstruktiven Maßnahmen konkurrieren metallische und biodegradierbare Implantate als Stabilisatoren. Beim Gebrauch auftragender metallischer Implantate ist darauf zu achten, dass die artikulierende Gelenkfläche ausgespart bleibt, da sonst Knorpelabrieb oder ein Impingement mit eingeschränkter Rotation auftreten. So ist seitlich am Radiuskopf nur ein schmaler Sektor im radial gelegenen Viertel bei Neutralstellung des Unterarms nicht bei der Pro- und Supination beteiligt. Diese „safe-zone“ nach Hotchkiss [7] erlaubt als einzige Stelle die Anlage von metallischen Implantaten. Auch die tellerförmige Gelenkfläche zum Humerus, die an der Beugung und Streckung beteiligt ist, sollte keine Stufen oder vorstehende Schraubenköpfe aufweisen.

Der Einsatz von biodegradierbaren Stiften zur Rekonstruktion des Radiuskopfs ermöglicht dagegen, die kleinen Stiftköpfe stufenfrei in den Knorpel zu versenken und somit weitere Platzierungspunkte an der Seite und von oben außerhalb der „safe-zone“ zu wählen.

Biodegradierbare Implantate eignen sich gut zur Fixierung kleiner osteochondraler Fragmente und ersparen dem Patienten durch vollständige Auflösung eine Metallentfernung. Im Idealfall wird eine Situation wie vor dem Unfall erreicht [20].

In der Unfallchirurgie der Universitätsklinik Köln werden seit über 10 Jahren biodegradierbare Poly-L/DL-Laktidstifte (Polypin ${ }^{\circledR}$, Fa. Centerpulse, Freiburg) für derartige Eingriffe eingesetzt. Einer Weiterentwicklung dieser Stifte wurden $10 \% \beta$-Trikalzium-Phosphat beigesetzt, um die Degradationsgeschwindigkeit etwas zu verlangsamen. Diese neuen Composite-Stifte wurden Polypin ${ }^{\circledR}$-C-Stifte genannt. Die In-vitro-Degradationseigenschaften wurden im Institut für unfallchirurgische Forschung und Biomechanik in Ulm (Direktor: Prof. Dr. L. Claes) getestet [9]. Nach einer tierexperimentellen Studie über 3 Jahre [19] wurden die neuen Stifte ab Ende 1996 in der Klinik eingesetzt. Das Projekt wurde vom BMFT, dem Verfügungsfond der Universität zu
Herrn Professor Dr. K. E. Rehm zum 60. Geburtstag gewidmet 
Köln, und der Imhoff-Stiftung in Köln finanziell unterstützt.

\section{Material und Methode}

In dieser prospektiven Untersuchung wurden Patienten eingeschlossen mit MasonII- und rekonstruierbaren Mason-IIIFrakturen des Radiuskopfs mit einer Gelenkstufe von über 2 Bildmillimetern oder Abkippung des Fragments von über $10^{\circ}$ auf dem präoperativen Röntgenbild in a.-p.- oder seitlicher Projektion oder auf einer Radiuskopfzielaufnahme.

Der Eingriff erfolgte in Oberarmblutleere über einen kurzen antero-radialen Zugang direkt über dem Radiuskopf. Das Lig. anulare wurde gespalten und nach Stabilisierung wieder genäht. Eine Ablösung des radialen Seitenbands erfolgte nur in 1 Fall.

Nach manueller Reposition wurde zur passageren Sicherung ein o,8-mm-Kirschner-Draht eingebracht. Divergierend wurden dann 2 2,0-mm-Löcher gebohrt und jeweils ein entsprechend gekürzter 2,0-mm-Polypin ${ }^{\circledR}$-C-Stift eingeschlagen.

Postoperativ sowie nach 3 und 6 Wochen wurden konventionelle Röntgenaufnahmen in 2 Ebenen angefertigt. Danach wurden die Patienten alle 6 Monate klinisch und radiologisch nachuntersucht. Von 29 Patienten konnten Computertomographien in 1-mm-Dünnschichttechnik angefertigt werden, an welchen die Frakturheilung, fortbestehende Stufen und Verkalkungen bewertet wurden. Die dargestellten Stiftkanäle wurden vermessen und auf Osteolysen und Degradation untersucht. Die Bewertung der Osteolysen erfolgte nach der Klassifikation von Hoffmann. Eine Aufweitung von über $1 \mathrm{~mm}$ um den initialen Stiftkanal wurde als Osteolyse Grad I und eine Aufweitung von über $3 \mathrm{~mm}$ als Osteolyse Grad II eingeschätzt [6] (• Abb. 1,2,3,4,5).

Das klinische Ergebnis wurde nach einem Score von Broberg u. Morrey [3] nach einer Modifikation von Geel et al. [5] bewertet ( $\bullet$ Tabelle 1): Im besten Fall waren 100 Punkte erreichbar. Ein sehr gutes Ergebnis wurde bei 90-10o Punkten, ein gutes bei 80-89 Punkten, ein befriedigendes bei 70-79 Punkten und ein

Trauma Berufskrankh 2004 - 6 [Suppl 3] : S344-S350

DOI 10.1007/s10039-003-0724-1

C Springer-Verlag 2003

\section{A.Prokop · A.Jubel · U.Hahn · K. E. Rehm}

\section{Speichenköpfchenbrüche: Wann soll wie operiert werden?}

\section{Zusammenfassung}

Mason-I-Speichenköpfchenfrakturen werden konservativ frühfunktionell durch Entlastung über 6 Wochen behandelt. Mason-II-Frakturen mit Dislokationen über $10^{\circ}$ oder einer Gelenkstufe von über 2 Bildmillimetern werden operiert. Biodegradierbare Implantate aus Polylaktid können unter den Knorpel versenkt und somit von der gesamten erreichbaren Gelenkfläche aus eingebracht werden. 35 Patienten wurden mit einem neuen Polylaktid-Composite-Stift stabilisiert und 34 nach durchschnittlich 38 Monaten nachuntersucht. 31 Patienten mit Mason-II-Frakturen wiesen im Mittel 96 von 100 möglichen Punkten im Broberg-Morrey-Score auf.Im CT wurden in 2 Fällen nach 18 und 24 Monaten passagere, erstgradige, asymptomatische Osteolysen beobachtet, die sich nach 6 Monaten vollständig zurückbildeten. Ab dem 2. Jahr waren die Stifte im konventionellen Röntgenbild nicht mehr sichtbar. Im CT waren über 5 Jahre eine stetige Implantatreduktion und Auffüllung der ehemaligen Stiftkanäle mit Knochen nachweisbar. Bei Trümmerfrakturen mit begleitender Halsfraktur sollte der Radiuskopf reseziert werden. Eine Radiuskopfprothese ist nur bei resultierender Instabilität notwendig und sollte dann so früh wie möglich implantiert werden.

\section{Schlüsselwörter}

Radiuskopffraktur · Biodegradierbare Implantate Poly- $\cdot$ L/DL $\cdot$-Laktid $\cdot$ Composite ·

Mason-Klassifikation

\section{Radial head fractures: which operation technique should be performed?}

\section{Abstract}

The Mason classification is used for radial head fractures. Mason type I fractures receive functional conservative treatment of no loading for 6 weeks. Mason II fractures with a displacement greater than $10^{\circ}$ or a depression of greater than $2 \mathrm{~mm}$ are treated by open reduction using biodegradable implants constructed of polylactide. These implants have the advantage of maintaining the joint surface by placement beneath the surface of the articular cartilage. Furthermore, the implants can be placed from various positions through the articular surface and are therefore more versatile than other modes of open reduction. Thirty-five patients were treated using the new polylactide composite pins (Polypin-C-pins). Average follow-up of 34 patients was 38 months: 31 with Mason type II fractures presented with a score of 96 out of 100 using the Broberg and
Morrey score. CT scans were performed after 18 and 24 months in all patients. One first-degree asymptomatic osteolysis was observed at 18 months, which was reduced at 24 months. Two years later the pins were not visible on conventional $X$-rays. $C T$ scan at 5 years revealed the implants at various levels of resorption and bony regrowth. In complex radial head fractures with involvement of the radial neck (Mason III), resection of the radial head is necessary. Radial head replacement is only required in cases of persistent instability. If replacement is required it should be done as soon as possible.

Keywords Radial head fracture · Biodegradable implants Poly-L/DL-lactide · Composite · Mason classification 


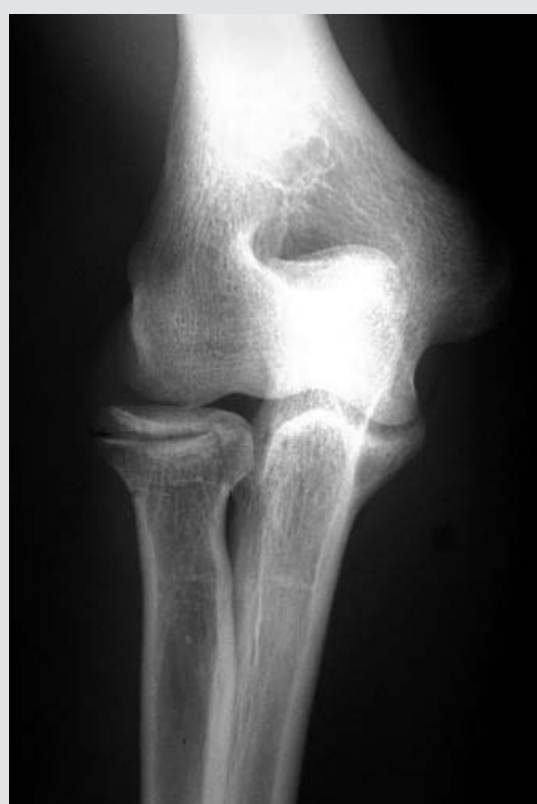

Abb. $1 \Delta$ Röntgenbild Unfall a.-p. mit Gelenkstufe von über 2 Bildmillimetern

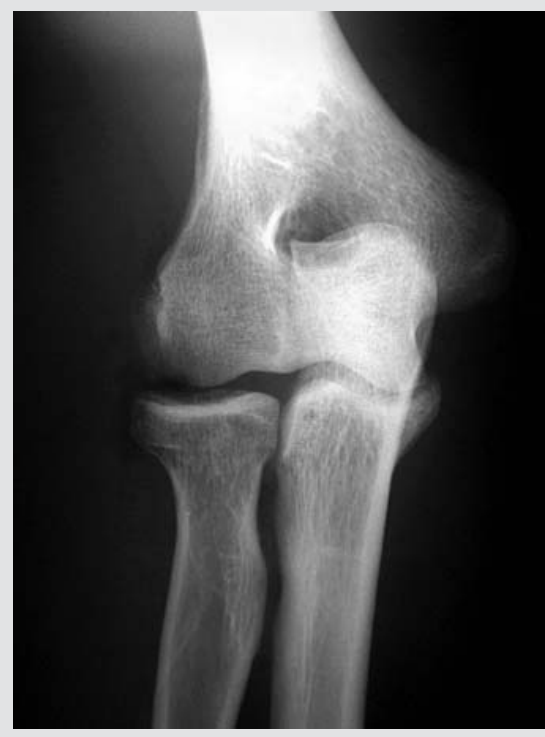

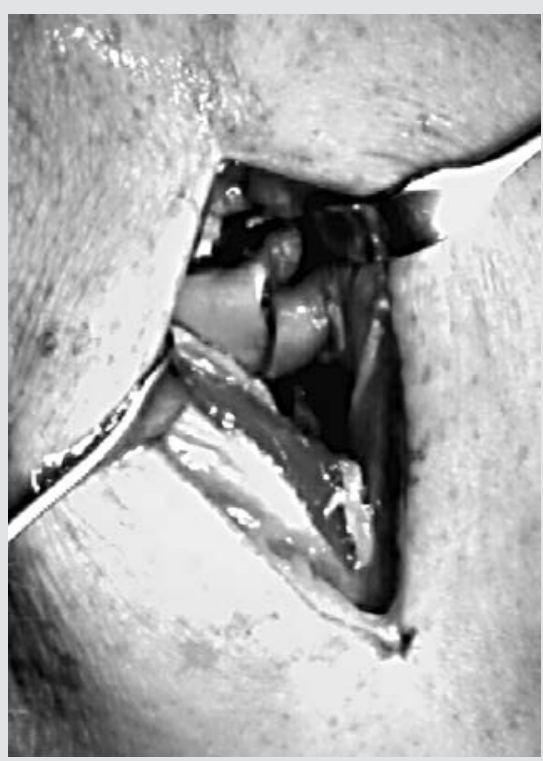

Abb. $2 \Delta$ Intraoperativer Situs mit deutlich disloziertem Gelenkfragment

Abb. $4<$ Röntgenbild nach 2 Jahren. Die Stifte haben sich bereits $z$. T resorbiert und sind auf dem konventionellen Röntgenbild nicht mehr sichtbar

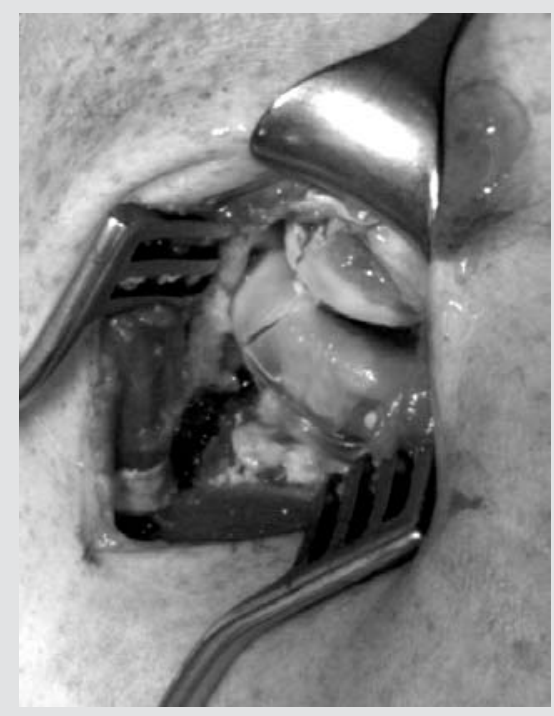

Abb. $3 \Delta$ Abschlusssitus mit stufenfreier Gelenkfläche und sichtbarem Polypin-C-Stift

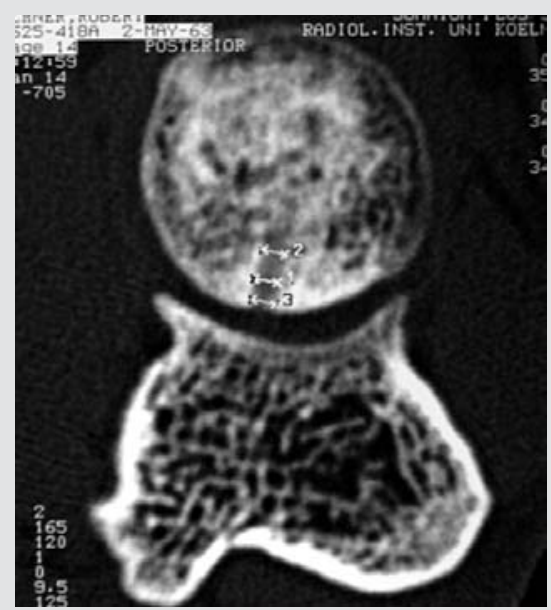

Abb. $5 \Delta$ CT sagittal nach 2 Jahren. Die Fraktur ist nicht mehr zu sehen. Es ist nur noch ein partieller Restkanal von einem Stift mit einem Durchmesser von $1,0 \mathrm{~mm}$ erkennbar schlechtes bei unter 70 Punkten erreicht $[3,5]$.

\section{Ergebnisse}

Vom 30.10.1996-1.4.2002 wurden $35 \mathrm{~Pa}$ tienten operiert. Es handelte sich um 22 Männer und 13 Frauen. In 19 Fällen war der linke und in 16 Fällen der rechte Arm betroffen.

Das mittlere Alter zum Unfallzeitpunkt betrug 42,3 Jahre (14-73 Jahre). Alle Patienten waren auf den ausgestreckten Arm gestürzt. Dabei waren 11 Patienten beim
Sport und hier zumeist beim Fahrradfahren (6-mal) verunfallt. In 4 Fällen handelte es sich um einen Arbeitsunfall.

Eine Typ-II-Fraktur nach Mason lag in 31 Fällen vor (CCF 21B2.1). Hier wurden im Mittel 2,5 Stifte pro Patient eingesetzt.

Bei 4 Patienten wurde eine Mason-IIIFraktur (CCF 21B2.2 und 21B2.3) behandelt. Hier wurden im Durchschnitt 3,75 Stifte pro Patient verwendet. In 2 Fällen wurden zusätzlich Mini-T-Platten und 1-mal ein TEN eingesetzt.

In 7 Fällen wurden intraoperativ begleitende Knorpelverletzungen (20\%) am
Epicondylus radialis beobachtet. Bei 3 Patienten wurden osteochondrale Frakturen ebenfalls mit Polypin ${ }^{\circledR}$-C-Stiften und 1-mal ein großes Fragment mit einer Schraube fixiert, 3 freie kleinere Knorpelfragmente wurden entfernt.

Die Eingriffe wurden von 12 verschiedenen Operateuren ausgeführt. Die durchschnittliche Schnitt-Naht-Zeit betrug 54 min. Intraoperativ kam es in 2 Fällen zum Abbruch des Stiftkopfs beim Einschlagen. Die Stifte konnten aber belassen werden. 
Tabelle 1

Borberg-Morrey-Score, modifiziert nach Geel [5]

\begin{tabular}{|c|c|c|c|}
\hline Untersucht & Variabel & Punkte & Gesamtpunkte \\
\hline Bewegung & $\begin{array}{l}\text { Beugung }\left[\left[^{\circ}\right] \times 0,2 \text { Punkte }\right. \\
\text { Streckung }\left[{ }^{\circ}\right] \times 0,2 \text { Punkte } \\
\text { Innenrotation }\left[{ }^{\circ}\right] \times 0,2 \text { Punkte } \\
\text { Außenrotation }\left[{ }^{\circ}\right] \times 0,2 \text { Punkte }\end{array}$ & $\begin{array}{l}\text { Maximal } 30 \text { Punkte } \\
\text { Maximal } 2 \text { Punkte } \\
\text { Maximal } 16 \text { Punkte } \\
\text { Maximal } 16 \text { Punkte }\end{array}$ & Maximal 64 Punkte \\
\hline Kraft & $\begin{array}{l}\text { Normal } \\
20 \% \text { Verlust } \\
\leq 50 \% \text { Verlust } \\
>50 \% \text { Verlust }\end{array}$ & $\begin{array}{l}12 \text { Punkte } \\
8 \text { Punkte } \\
6 \text { Punkte } \\
\text { 0 Punkte }\end{array}$ & Maximal 12 Punkte \\
\hline Stabilität & $\begin{array}{l}\text { Normal } \\
\text { Gering ohne Einschränkung } \\
\text { Instabil }\end{array}$ & $\begin{array}{l}12 \text { Pkt } \\
6 \text { Punkte } \\
\text { 0 Punkte }\end{array}$ & Maximal 12 Punkte \\
\hline Schmerzen & $\begin{array}{l}\text { Keine } \\
\text { Mild (keine Analgetika, normale Aktivität) } \\
\text { Moderat (bei oder nach Aktivität) } \\
\text { Stark (ohne Belastung, in Ruhe) }\end{array}$ & $\begin{array}{l}12 \text { Punkte } \\
8 \text { Punkte } \\
6 \text { Punkte } \\
0 \text { Punkte }\end{array}$ & Maximal 12 Punkte \\
\hline Gesamt & & & Maximal 100 Punkte \\
\hline
\end{tabular}

Postoperativ traten keine Wundheilungsstörungen oder Infektionen auf. Alle Patienten wurden funktionell nachbehandelt. Nach 6 Wochen erfolgte die Vollbelastung. Die durchschnittliche Arbeitsunfähigkeit betrug 4,6 Wochen (o-12 Wochen). Sportfähigkeit wurde nach im Mittel 14,4 Wochen (1-70 Wochen) erreicht.

Ein Patient verstarb 6,5 Monate nach der Operation an den Folgen eines metastasierenden Brochialkarzinoms.

Zwischen dem 1.3.2002 und dem 15.5.2002 wurden 34 der 35 Patienten $(97,1 \%)$ klinisch und konventionell radiologisch noch einmal untersucht. In 29 Fällen wurde zusätzlich ein CT durchgeführt. Der durchschnittliche Zeitraum zwischen Operation und Nachuntersuchung betrug 38,2 Monate (1,5-64,5 Monate).

Die Narben waren bei der Untersuchung reizlos verheilt, und die Gelenke wiesen keinen Erguss auf. Der BrobergScore betrug bei allen nachuntersuchten Patienten im Mittel 96 Punkte (67100 Punkte) nach 38,2 Monaten. 31 Patienten wiesen exzellente Ergebnisse auf, 2-mal wurden gute und 1-mal ein schlechtes Ergebnisse erreicht (• Tabelle 2). Die durchschnittliche Bewegungseinschränkung bei Innen- und Außenrotation betrug $8,1^{\circ}$ und bei Beugung und Streckung $9,5^{\circ}$ im Vergleich zur unverletzten Gegenseite.
Bei dem Fall mit einem schlechten Ergebnis handelte es sich um einen 70-jährigen Patienten mit einer Mason-II-Fraktur, die zwar gut verheilt war, aber zu einer überschießenden periartikulären Verkalkung des proximalen Radioulnargelenks mit Ankylose geführt hatte. Eine Arthrolyse wurde vom Patienten nicht gewünscht.

Auf den konventionellen Röntgenbildern des Ellenbogens und den CT-Bildern waren bei der Nachuntersuchung keine Osteolysen nachweisbar. 2-mal wurden vor der Nachuntersuchung bei CT-Kontrollen nach 18 und 24 Monaten asymptomatische erstgradige Osteolysen um einen Stiftkopf gesehen, die bei Kontrollen bereits 6 Monate später wieder verschwunden waren. In den CT-Untersuchungen zeigte sich eine beginnedne Auflösung der Stifte ab dem 12.-18. Monat. $\mathrm{Ab}$ dem 24. Monat verkleinerte sich das Restvolumen der Stifte im CT, und im konventionellen Röntgenbild waren sie nicht mehr sichtbar. Nur im CT waren unregelmäßig begrenzt vereinzelte Randsklerosierungen um die ehemaligen Stiftkanäle nachweisbar. In den Kanälen war die gleiche Knochendichte wie von spongiösem Knochen messbar (• Abb. 1,2,3,4,5).

\section{Diskussion}

Für das Ellenbogengelenk gelten heutzutage die gleichen Grundsätze wie für alle anderen Gelenkfrakturen, dass eine anatomische Rekonstruktion mit möglichst stufenfreier Gelenkfläche anzustreben ist. So wird der unverschobene Mason-IBruch mit sehr guten Ergebnissen konservativ frühfunktionell durch kurzfristige Ruhigstellung bis zur Schmerzfreiheit (maximal 1 Woche) und dann Entlastung für 6 Wochen behandelt. Regelmäßige Röntgenkontrollen sind zur Stellungskontrolle notwendig. Bei den sehr seltenen sekundären Dislokationen sollte frühzeitig operiert werden [16]. Wir haben 50 Patienten mit Mason-I-Frakturen auf diese Weise behandelt und nach 12 Monaten bei einer Nachuntersuchung einen durchschnittlichen Broberg-Score von 99 Punkten erreichen können.

Mason-II-Frakturen mit Gelenkstufen von über 2 Bildmillimetern oder einer Achsenabkippung von über $10^{\circ}$ werden operativ behandelt. Um die genaue Dislokation beurteilen zu können, sollte neben der a.-p. und seitlichen Röntgenaufnahme auch eine Radiuskopfzielaufnahmen $\left(45^{\circ}\right.$ seitlich, Strahlengang von lateral nach medial) vorgenommen werden.

Zur Versorgungen werden in der Literatur überwiegend Schrauben und $\mathrm{Ab}$ - 


\begin{tabular}{|c|c|c|c|c|c|c|c|c|c|c|c|c|c|}
\hline Nr. & $\begin{array}{l}\text { Pa- } \\
\text { tient }\end{array}$ & $\mathrm{m} / \mathrm{w}$ & Alter & Pins & Seite & Mason & $\begin{array}{l}\text { Arbeits- } \\
\text { unfähig } \\
\text { [Wochen] }\end{array}$ & $\begin{array}{l}\text { Sport- } \\
\text { unfähig } \\
\text { [Wochen] }\end{array}$ & $\begin{array}{l}\text { Nachunter- } \\
\text { suchung } \\
\text { [Monate] }\end{array}$ & $\begin{array}{l}\text { Extension/ } \\
\text { Flexion bei } \\
\text { Nachunter- } \\
\text { suchung }\end{array}$ & $\begin{array}{l}\text { Außenrotation/ } \\
\text { Innenrotation } \\
\text { bei Nachunter- } \\
\text { suchung }\end{array}$ & $\begin{array}{l}\text { CT Rest- } \\
\text { lumen } \\
{[\%]}\end{array}$ & $\begin{array}{l}\text { Broberg- } \\
\text { Score }\end{array}$ \\
\hline 1 & PB & w & 41 & 3 & Links & II & 3 & 12 & 57,3 & $10-0-150$ & $90-0-90$ & 20 & 100 \\
\hline 2 & NA & $\mathrm{m}$ & 41 & 2 & Links & II & 3 & 6 & 60,1 & $10-0-140$ & $80-0-80$ & 0 & 98 \\
\hline 3 & HU & w & 27 & 2 & Rechts & II & 8 & 24 & 60,5 & $10-0-150$ & $90-0-90$ & 25 & 100 \\
\hline 4 & GM & $\mathrm{m}$ & 24 & 2 & Rechts & II & 2 & 6 & 61,9 & $5-0-150$ & $85-0-90$ & 20 & 100 \\
\hline 5 & SM & $\mathrm{m}$ & 62 & 6 & Links & III & 3 & 4 & 63,2 & $5-0-150$ & $90-0-90$ & 30 & 99 \\
\hline 6 & SP & $\mathrm{m}$ & 70 & 4 & Links & II & 1 & 70 & 58,7 & $0-10-120$ & $20-0-20$ & 25 & 67 \\
\hline 7 & LE & $w$ & 51 & 3 & Links & II & 6 & 16 & 64,5 & $0-10-150$ & $90-0-90$ & 15 & 96 \\
\hline 8 & FR & $\mathrm{m}$ & 34 & 2 & Rechts & II & 4 & 12 & 53,7 & $5-0-140$ & $85-0-90$ & 20 & 98 \\
\hline 9 & NM & w & 31 & 3 & Rechts & II & 5 & 24 & 53,1 & $0-0-150$ & $90-0-80$ & 25 & 97 \\
\hline 10 & $\mathrm{NE}$ & w & 68 & 2 & Links & II & 6 & 24 & 53,4 & $0-10-140$ & $90-0-90$ & 15 & 96 \\
\hline 11 & KH & $\mathrm{m}$ & 73 & 3 & Rechts & III & - & - & Verstorben & - & - & - & Tod \\
\hline 12 & PT & $\mathrm{m}$ & 41 & 3 & Rechts & II & 12 & 16 & 12,6 & $10-0-150$ & $90-0-90$ & 70 & 100 \\
\hline 13 & LD & $\mathrm{m}$ & 55 & 2 & Links & II & 2 & 8 & 51,8 & $5-0-150$ & $90-0-90$ & & 96 \\
\hline 14 & SH & $\mathrm{m}$ & 14 & 4 & Rechts & II & 1 & 6 & 46,8 & $0-15-150$ & $50-0-60$ & 20 & 84 \\
\hline 15 & HU & $w$ & 33 & 2 & Links & II & 4 & 4 & 47,4 & $10-0-150$ & $90-0-90$ & 25 & 100 \\
\hline 16 & KK & w & 62 & 2 & Rechts & II & 6 & 8 & 44,8 & $0-10-150$ & $90-0-90$ & 40 & 96 \\
\hline 17 & NM & $\mathrm{m}$ & 45 & 4 & Links & II & 0 & 1 & 43,8 & $0-10-145$ & $90-0-90$ & - & 97 \\
\hline 18 & BK & $\mathrm{m}$ & 38 & 2 & Rechts & II & 1 & 16 & 42,6 & $0-10-150$ & $90-0-90$ & 45 & 97 \\
\hline 19 & GC & w & 56 & 2 & Rechts & II & 4 & 5 & 42,6 & $10-0-150$ & $90-0-90$ & 50 & 100 \\
\hline 20 & $H R$ & $\mathrm{~m}$ & 57 & 2 & Links & II & 6 & 52 & 41 & $0-0-150$ & $60-0-90$ & 50 & 94 \\
\hline 21 & FC & w & 24 & 2 & Links & II & 3 & 8 & 41,7 & $10-0-150$ & $90-0-90$ & 50 & 100 \\
\hline 22 & WM & w & 40 & 4 & Links & II & 12 & 36 & 38,7 & $0-10-150$ & $90-0-80$ & 60 & 97 \\
\hline 23 & so & $\mathrm{m}$ & 33 & 2 & Rechts & II & 4 & 14 & 32,3 & $5-0-150$ & $90-0-90$ & 55 & 99 \\
\hline 24 & MM & w & 63 & 4 & Rechts & II & 5 & 12 & 29 & $0-0-150$ & $90-0-90$ & 70 & 99 \\
\hline 25 & $\mathrm{FF}$ & $\mathrm{m}$ & 30 & 3 & Links & III & 4 & 8 & 9,2 & $0-0-150$ & $90-0-90$ & 80 & 99 \\
\hline 26 & FP & $\mathrm{m}$ & 69 & 3 & Links & II & 8 & 12 & 22,2 & $0-0-150$ & $50-0-50$ & 25 & 88 \\
\hline 27 & TG & $\mathrm{m}$ & 55 & 2 & Rechts & II & 6 & 10 & 20,6 & $0-0-150$ & $90-0-90$ & 50 & 100 \\
\hline 28 & $A M$ & $\mathrm{~m}$ & 39 & 2 & Links & II & 7 & 8 & 17,7 & $10-0-150$ & $90-0-90$ & 50 & 100 \\
\hline 29 & BM & $\mathrm{m}$ & 32 & 2 & Links & II & 6 & 14 & 17,4 & $10-0-150$ & $90-0-90$ & 55 & 100 \\
\hline 30 & $\mathrm{DH}$ & w & 45 & 2 & Links & II & 6 & 8 & 12,6 & $0-0-150$ & $80-0-80$ & 55 & 98 \\
\hline 31 & KE & $\mathrm{m}$ & 52 & 2 & Links & II & 2 & 2 & 6,6 & $5-0-150$ & $90-0-90$ & 75 & 100 \\
\hline 32 & MM & $w$ & 33 & 2 & Rechts & II & 3 & 6 & 7,2 & $5-0-150$ & $90-0-90$ & 80 & 99 \\
\hline 33 & RM & $\mathrm{m}$ & 22 & 3 & Rechts & II & 3 & 16 & 4,7 & $0-0-135$ & $90-0-90$ & 100 & 97 \\
\hline 34 & HT & $\mathrm{m}$ & 38 & 3 & Rechts & III & 6 & 8 & 1,6 & $0-0-140$ & $80-0-80$ & - & 96 \\
\hline 35 & WA & $w$ & 32 & 2 & Links & II & 6 & - & 1,4 & $0-0-150$ & $90-0-90$ & - & 94 \\
\hline
\end{tabular}

stützplatten eingesetzt $[2,7]$ und die Implantate, wenn nicht aus Titan, zumeist auch wieder entfernt. Die Kosten der Metallentfernung, die heute in einem Krankenhaus der 4. Versorgungsstufe bei einem 1- bis 3-tägigen Aufenthalt anfallen, liegen im günstigsten Fall (Tagessatz $250 €$ ) bei 250-750 € Hinzu kommt bei einer Arbeitsunfähigkeit von 2 Wochen ein betriebswirtschaftlicher Verlust von zusätzlich etwa $6000 €[15]$. Metallische Implantate haben zwar den unbestreitbaren Vorteil höherer Stabilität, die aber am Radi- uskopf, als gering belastete Zone, nicht erforderlich ist.

Zur Vermeidung von Folgeeingriffen wurden in den 6oer Jahren von Kulkarni et al. [12] selbstauflösende, biodegradierbare Implantate aus Polylaktid und anderen Polymeren entwickelt. Ein wesentlicher Vorteil biodegradierbarer Implantate ist die kontinuierliche Abnahme an biomechanischer Festigkeit des Implantats spiegelbildlich zum Aufbau des heilenden Knochens, an den die tragende Funktion dynamisch zurückgegeben wird. Im
Gegensatz dazu fällt beim Stahlimplantat die Gesamtfestigkeit der Konstruktion bei der Metallentfernung abrupt auf das Niveau des bis dahin geheilten Knochens ab (stress-protection).

Bei den degradierbaren Materialien ist die Biokompatibilität dosisabhängig [8]. Am Radiusköpfchen ist die Implantatmenge mit 2-3 Stiften immer gering. Alle Arbeitsgruppen, die mit biodegradierbaren Implantaten Erfahrung haben, empfehlen daher deren Einsatz bei der Radiuskopffraktur [18, 22]. 
Allerdings mussten anfänglich, besonders bei Polyglykolidstiften, erhöhte Komplikationsraten hingenommen werden, die durch Knochenresorption und aseptische Fistelbildung gekennzeichnet waren [23]. Bei den langsamer degradierbaren Polylaktidimplantaten (Polypin ${ }^{\circledR}$ ) wurden diese Reaktionen nur sehr vereinzelt beschrieben.

Marzischewski et al. [17] berichteten über Erfahrungen in der Zeit von Mai 1990-März 1996. In diesem Zeitraum wurden bei 97 Patienten Polypin ${ }^{\circledR}$-Stifte eingesetzt. Um über eine genügend lange Nachbeobachtungszeit zu berichten, wurden 34 Patienten nach 22-54 Monaten kontrolliert und die Ergebnisse ausgewertet. In keinem Fall war es zu Weichteil- oder Fremdkörperreaktionen gekommen. In 6 Fällen wurde eine asymptomatische erstgradige Osteolyse im konventionellen Röntgenbild festgestellt. $33 \mathrm{~Pa}$ tienten beurteilten das Ergebnis bei der Nachuntersuchung als gut und sehr gut [17].

Rehm et al. [21] publizierten 1999 die einzige prospektiv randomisierte Multizenterstudie bei Radiuskopffrakturen. In dieser Studie wurden Radiuskopfmeißelfrakturen (CCF 21B2) entweder mit Metallschrauben oder mit Polypin ${ }^{\circledR}$ stabilisiert. Indikation zur Operation waren eine Stufe von mindestens $2 \mathrm{~mm}$ oder eine Dislokation von $10^{\circ}$ auf dem Röntgenbild. 165 Patienten wurden in die Untersuchung einbezogen, davon 82 Patienten, bei denen Metallschrauben, und 83 Patienten, bei denen Polypin ${ }^{\circledR}$-Stifte eingesetzt wurden. Postoperativ kam es in 1 Fall in der Polypin ${ }^{\circledR}$-Gruppe zu einer tiefen Wundinfektion. Fremdkörperreaktionen, Serome oder Fisteln traten in beiden Gruppen nicht auf. Eine Osteolyse mit Störung der Frakturheilung wurde in der Polypin ${ }^{\circledR}$ Gruppe 2-mal und in der Metallgruppe 1-mal festgestellt. 92\% der Patienten $(n=151)$ konnten in den ersten 6 Wochen nachuntersucht werden, der BrobergScore betrug in der Metallgruppe 74 und in der Polypin ${ }^{\circledR}$-Gruppe 77 Punkte. Zwischen dem 9. und 12. Monat wurden $84 \%$ der Patienten $(n=138)$ kontrolliert, wobei der Broberg-Score sowohl in der Metallals auch in der Polypin ${ }^{\circledR}$-Gruppe 91 Punkte betrug. Nach 2 Jahren konnten $82 \%$ der Patienten $(n=135)$ untersucht werden.
Sie erreichten einen Broberg-Score von 91 Punkten in der Metallgruppe und 93 Punkten in der Polypin ${ }^{\circledR}$-Gruppe.Zwischen den beiden Gruppen bestand kein statistisch signifikanter Unterschied [21].

Mit den weiterentwickelten Polypin ${ }^{\circledR}$ C-Stiften wurde in der eigenen Untersuchung nach durchschnittlich 38 Monaten sogar ein Broberg-Score von 96 Punkten erreicht.

Lindemann-Sperfeld et al. [13] untersuchten 26 Patienten mit Mason-II-Frakturen, die mit jeweils 2 2,7-mm-Minischrauben behandelt wurden. Nach durchschnittlich 2,2 Jahren wurden die Patienten nachuntersucht. Nur 7 Patienten (27\%) erreichten nach dem Broberg-Score ausgezeichnete Ergebnisse. 10 Patienten erzielten gute und 8 Patienten sogar nur befriedigende Ergebnisse. Ein Patient wies einen Scorewert von unter 60 Punkten auf [13].

Khalfayan et al. [11] berichteten 1992 über 26 Patienten mit Mason-II-Frakturen, die nach durchschnittlich 18 Monaten nachuntersucht wurden.10 Patienten wurden mit Herbert- oder Minischrauben und 16 konservativ frühfunktionell versorgt. Nach der Operation wurde ein Broberg-Score von im Mittel 92 Punkten und bei der konservativen Behandlung nur von 77 Punkten erreicht [11].

Demnach bietet die operative Versorgung der Mason-II-Fraktur signifikante Vorteile gegenüber dem konservativen Vorgehen. Beim Vergleich der verschiedenen Operationsverfahren werden mit dem Polypin ${ }^{\circledR}-\mathrm{C}$-Stift die besten Scorewerte erreicht.

Während sich auch Mehrfragmentfrakturen gut mit Pins aufbauen lassen, bleibt die Halsfraktur eine Schwachstelle. Bei Mehrfragmentfrakturen mit Ablösung vom Hals (Mason III) ist die Rekonstruktion der Radiuskopfs sehr anspruchsvoll und nur in wenigen ausgesuchten Fällen erfolgreich. Begleitende T-Platten müssen in der gering artikulierenden „safe-zone“ angebracht werden, stören aber häufig trotzdem die Umwendbewegung [7]. Intramedullär von distal eingeführte Prévot-Stifte können die Fraktur zwar aufrichten, sich aber nur selten stabil im Kopf verspannen.

In der Literatur wird bei Mason-IIIVerletzung die primäre Resektion emp- fohlen. Lindemann-Sperfeld et al. [13] berichteten über 31 Fälle nach Resektion bei Mason-III-Frakturen. In 54,6\% der nach 2-5 Jahren untersuchten Patienten konnten so gute und sehr gute Ergebnisse im Broberg-Score erzielt werden. 24,2\% der Patienten wiesen befriedigende und 21,2\% schlechte Resultate auf [13].

Der prothetische Ersatz nach Resektion des Radiuskopfs ist nur dann notwendig, wenn nach Resektion eine Instabilität des Ellenbogens verbleibt. Dann sollte die Prothese primär eingebracht werden. Wick et al. [24] konnten $30 \mathrm{~Pa}$ tienten nach prothetischem Ersatz nach durchschnittlich 8,1 Jahren nachuntersuchen. Nur $27 \%$ der Patienten erreichten ein gutes, $47 \%$ ein befriedigendes und $27 \%$ ein schlechtes Ergebnis.

Sekundär implantierte Prothesen führen zu schlechteren Ergebnissen. So konnten Ambacher et al. [1] 33 Patienten nach Prothesen nach durchschnittlich 37 Monaten untersuchen. Von den primär implantierten Radiuskopfprothesen wiesen 10 von 14 Patienten gute und mäßige und 4 von 14 schlechte Ergebnisse auf. Bei den Sekundärimplantationen wurden nur bei 4 von19 Patienten gute und mäßige und bei 15 von 19 schlechte Ergebnisse beobachtet [1].

Bei den hier untersuchten Radiuskopffrakturen haben die eingesetzten biodegradierbaren Polypin ${ }^{\circledR}$-C-Stifte in allen Fällen zur knöchernen Ausheilung der Frakturen bei guten klinischen Ergebnissen geführt.

Die langsame Auflösung der Stifte konnte auf den konventionellen Röntgenaufnahmen nachvollzogen werden. Zwischen dem 12. und 18. Monat waren die Röntgenmarkierungen nur noch fragmentiert und zwischen dem 24. und 36. Monat gar nicht mehr nachweisbar.

Die Darstellung der Stiftkanäle war dann nur noch im CT möglich. Die Kanäle wurden kleiner und unregelmäßiger. Im ehemaligen Implantatlager konnte CTmorphologisch die gleiche Dichte wie von spongiösem Knochen gemessen werden. Zurück blieb z. T. ein im CT noch sichtbarer kleiner unregelmäßiger sklerotischer Randsaum, obwohl das Implantat verschwunden war. 


\section{Fazit für die Praxis}

Mason-I-Frakturen werden mit sehr guten Ergebnissen frühfunktionell konservativ therapiert. Bei Gelenkstufen von über 2 Bildmillimetern oder Abkippungen von über $10^{\circ}$ (Mason II) sollte eine Rekonstruktion erfolgen. Dafür eignen sich biodegradierbare Polylaktidstifte besonders gut, da sie unter die Knorpeloberfläche versenkt und auch außerhalb der ,safe-zone" eingebracht werden können. Die Stifte werden ohne Folgeeingriff vollständig resorbiert und die Implantatlager mit Knochengewebe ersetzt. Bei Trümmerfrakturen mit begleitender Halsfraktur (Mason III) sollte der Radiuskopf reseziert werden. Nur bei Instabilität des Ellenbogens sollte, wenn möglich primär, eine Radiuskopfprothese implantiert werden.

\section{Korrespondierender Autor Priv.-Doz.Dr. A. Prokop}

Klinik und Poliklinik für Unfall-, Hand- und Wiederherstellungschirurgie, Klinikum der Universität zu Köln, Joseph-Stelzmann-Straße 9,50924 Köln, E-Mail: axel.prokop@uni-koeln.de

\section{Literatur}

1. Ambacher T, Maurer F, Weise K (2000) Behandlungsergebnisse nach primärer und sekundärer Radiusköpfchenresektion. Unfallchirurg 103: 437-443

2. Berreux P, Pelet D, Albrecht HU, Kunz H (1981) Die isolierten Radiusköpfchenfrakturen. Konservative und operative Therapie. Orthopäde 10: 297-302

3. Broberg MA, Morrey BF (1986) Results of delayed excision of the radial head after fracture.J Bone Joint Surg Am 68-A: 669-674

4. Frerichmann U, Schult M, Schiedel F, Joist A (2002) Die operative Versorgung von Radiuskopffrakturen. Hefte Unfallchirurg 284: 452-453

5. Geel C, Palmer A, Ruedi T, Leutenegger A (1990) Internal fixation of proximal radial head fractures.J Orthop Trauma 4: 270-274

6. Hoffmann R, Weiler A, Helling HJ, Krettek C, Rehm KE (1997) Lokale Fremdkörperreaktionen auf biodegradierbare Implantate. Unfallchirurg 100: 658-666

7. Hotchkiss RN (1997) Displaced fractures of the radial head: internal fixation or excision? J Am Acad Orthop Surg 5: 1-10

8. Ignatius AA, Claes LE (1996) In vitro biocompatibility of bioresorbable polymers: poly (L, DL-lactide) and poly (L-lactide-co-glycolide). Biomaterials 17: 831-839

9. Ignatius $A A$, Augat $P$, Claes LE (2001) Degradation behavior of composite pins made of tricalcium phosphate and poly(L,DL-lactide) for the reduction of small bony fragments. J Biomat Sci Polymer Edn 12: 185-194

10. Josten C, Korner J (2000) Frakturen und Luxationen am proximalen Unterarm. Trauma Berufskrankh [Suppl 2] 2: 194-198

11. Khalfayan EE, Culp RW, Alexander AH (1992) Mason type II radial head fractures: operative versus nonoperative treatment. J Orthop Trauma 6: 283-289
12. Kulkarni RK, Pani KC, Neumann C, Leonhard F (1966) Polylactid acid for surgical implants. Arch Surg 93: 839-843

13. Lindemann-Sperfeld L, Haferkorn K, Genest M, Jansch L, Martinschev I, Otto W (2000) Differenzialtherapie der Radiusköpfchenfraktur in Abhängigkeit vom Frakturtyp. Trauma Berufskrankh 2: 304-312

14. Lindemann Sperfeld L, Jansch L, Marintschev I, Otto W (2002) Differenziertes Behandlungskonzept der Radiusköpfchenfraktur in Abhängigkeit vom Frakturtyp. Hefte Unfallchirurg 284: 207-208

15. Lob G (1993) State of the art: biodegradable implantate. Hefte Unfallheilkd 232: 464-472

16. Mason ML (1954) Some observations on fractures of the head of the radius with a review of one hundred cases. Br J Surg 42: 123-132

17. Marzischewski S, Helling HJ, Rehm KE (1998) Klinische Langzeitergebnisse der Pilotanwendung neuer Polylactidstifte. Gibt es ein Biokompatibilitätsproblem? Hefte Unfallchirurg 265: 252-257

18. Pelto K, Hirvensalo E, Böstmann O, Rokkanen P (1994) Treatment of radial head fractures with absorbable polyglycolide pins: a study on the security of the fixation in 38 cases. J Orthop Trauma 8: 94-98

19. Prokop A (1999) Polylactid und Tricalciumphosphat. Verbesserung biodegradabler Implantate im Verbund. Eine vergleichende tierexperimentelle und klinische Untersuchung. Habilitationsschrift, Medizinische Fakultät, Universität zu Köln

20. Rehm KE, Helling HJ, Gatzka C (1997) Neue Entwicklungen beim Einsatz resorbierbarer Implantate. Orthopäde 26: $489-497$

21. Rehm KE, Helling HJ, Nagel M, Lilienthal J (1999) Displaced radial head fracture. Metal versus biodegradable pin fixation. $66^{\text {th }}$ Meeting AAOS, Annual Meeting, Anaheim, USA, Paper Presentation No.66

22. Van der Elst M, Patka P, Van der Werken C (2000) Resorbierbare Implantate für Frakturfixierung. Aktueller Stand. Unfallchirurg 103: 178-182

23. Weiler A, Helling HJ, Kirch U, Zirbes TK, Rehm KE (1996) Foreign-body reaction and the corse of osteolysis after polyglycolide implants for fracture fixation.J Bone Joint Surg Br 78B: 369-376

24. Wick M, Lies A, Müller EJ, Hahn MP, Muhr G (1998) Speichenköpfchenprothesen. Welche Ergebnisse sind zu erwarten? Unfallchirurg 101: 817-821 